\title{
Atividade inseticida do extrato das folhas de Piper hispidum (Piperaceae) sobre a broca-do-café (Hypothenemus hampei)
}

\author{
MAURÍCIO REGINADO ALVES DOS SANTOS ${ }^{1,3}$, ANDRINA GUIMARÃES SILVA ${ }^{1}$, \\ RENATO ABREU LIMA ${ }^{1}$, DANIELLA KARINE SOUZA LIMA ${ }^{1}$, \\ LUNALVA AURÉLIO PEDROSO SALLET ${ }^{1}$, CÉSAR AUGUSTO DOMINGUES TEIXEIRA ${ }^{1}$, \\ ALINE ROBERTA POLLI ${ }^{2}$ e VALDIR ALVES FACUNDO ${ }^{2}$
}

(recebido: 24 de abril de 2008; aceito: 01 de abril de 2010)

\begin{abstract}
Inseticidal activity of Piper hispidum (Piperaceae) leaves extract on (Hypothenemus hampei)). The objective of this work was to evaluate the insecticidal and repellent potential of the acetonic leaf extract of Piper hispidum Kunth on Hypothenemus hampei (Ferrari, 1867) insects by topical application, contaminated surface and repellent effect. Dilutions of $25.0 ; 5.0 ; 0.1 ; 0.02 ; 0.004$ and $0.0008 \mathrm{mg} \mathrm{mL}^{-1}$ were used for exposition in contaminated surface and topical application. In the repellence test $0.5 \mathrm{mg} \mathrm{mL}^{-1}$ of this extract were used. Evaluations occurred during 48 hours after exposure to the extract. In the exposition in contaminated surface $100 \%$ of mortality were observed in the dilution of $25.0 \mathrm{mg} \mathrm{mL}^{-1}$ and 50 to $80 \%$ in dilutions of 5.0 to $0.004 \mathrm{mg} \mathrm{mL}^{-1}$, while $0.0008 \mathrm{mg} \mathrm{mL}^{-1}$ and the control resulted in only $5 \%$ of mortality. In the topic application 60 to $65 \%$ of mortality were observed with dilutions of 25.0 to $0.1 \mathrm{mg} \mathrm{mL}^{-1}$. Dilutions of 0.0008 to $0.2 \mathrm{mg} \mathrm{mL}^{-1}$ did not differ from the control. The repellence index was lower than the minimum value praised in literature to consider a substance as repellent. These results pointed out to the high potential of $P$. hispidum extract for the control of H. hampei.
\end{abstract}

Key words - Coffea arabica, natural insecticide, Piperaceae

RESUMO - (Atividade inseticida do extrato das folhas de Piper hispidum (Piperaceae) sobre a broca-do-café (Hypothenemus hampei)). Este trabalho teve como objetivo avaliar o potencial inseticida do extrato acetônico das folhas de Piper hispidum Kunth sobre Hypothenemus hampei (Ferrari, 1867) em aplicação tópica, superfície contaminada e efeito de repelência. Adotou-se as diluições de 25,$0 ; 5,0 ; 0,1 ; 0,02 ; 0,004$ e $0,0008 \mathrm{mg} \mathrm{mL}^{-1}$ para exposição em superfície contaminada e aplicação tópica. No teste de repelência, utilizou-se a diluição $0,5 \mathrm{mg} \mathrm{mL}^{-1}$ do extrato. As avaliações foram realizadas nas 48 horas após a exposição ao extrato. Na exposição em superfície contaminada, obteve-se $100 \%$ de mortalidade, na diluição $25,0 \mathrm{mg} \mathrm{mL}^{-1}$, e de 50 a $80 \%$ nas diluições 0,004 a $5,0 \mathrm{mg} \mathrm{mL}^{-1}$, enquanto $0,0008 \mathrm{mg} \mathrm{mL}^{-1}$ e o controle resultaram em apenas $5 \%$ de mortalidade. Na aplicação tópica, atingiu-se 60 a $65 \%$ de mortalidade, com as diluições de 0,1 a $25,0 \mathrm{mg} \mathrm{mL}^{-1}$. As diluições de 0,2 a $0,0008 \mathrm{mg} \mathrm{mL}^{-1}$ não diferiram do controle. Os índices de repelência foram inferiores ao valor mínimo preconizado na literatura para se considerar uma substância como repelente. Este estudo evidencia a atividade inseticida do extrato de folhas de P. hispidum sobre H. hampei, o que sugere seu potencial no controle deste inseto.

Palavras-chave - Coffea arabica, inseticida natural, Piperaceae

\section{Introdução}

O café é o segundo produto mais comercializado do mundo, depois do petróleo. O Brasil é o maior produtor e exportador mundial de grãos de café (sendo também o segundo maior consumidor) (Bandeira et al. 2009) e é responsável por cerca de $30 \%$ da produção mundial (Resende et al. 2001). Historicamente, esta cultura tornou-se o principal produto econômico do país, promovendo o desenvolvimento agrícola do Sul e Sudeste brasileiro,

1. Embrapa Rondônia, Caixa Postal 406, 78900-970 Porto Velho, RO, Brasil.

2. Universidade Federal de Rondônia, Departamento de Química, BR 364, km 9,5, 78900-050 Porto Velho, RO, Brasil.

3. Autor para correspondência: mauricio@cpafro.embrapa.br e se classifica entre os primeiros gêneros considerados primários no comércio internacional (Tassaro 2008).

O agronegócio do café concentra uma das mais dinâmicas cadeias de produção brasileiras, com vantagens altamente competitivas: reputação e tradição dos exportadores, grande consumo no mercado interno, perfil tecnológico avançado das empresas líderes e refinamento do mercado financeiro e de capitais (Vegro et al. 2005), além de ocupar o sexto lugar na geração de empregos no país (Najberg \& Pereira 2004).

Em nível mundial, a cultura é extremamente fragmentada, com $20 \%$ da colheita proveniente de grandes plantações e o restante dividido entre pequenas propriedades, e representa a fonte de subsistência para cinco milhões de famílias no mundo, que cultivam em superfícies de cinco hectares ou menos. Para muitos 
pequenos produtores, que vivem também da colheita de uma agricultura de subsistência, o café representa o único produto vendável no mercado, porque os demais cultivos destinam-se ao autoconsumo (Eccardi \& Sandalj 2000).

Estima-se que a broca-do-café, Hypothenemus hampei (Ferrari, 1867) - Coleoptera: Scolytidae, provoque danos da ordem de 500 milhões de dólares em todo o mundo. O controle da praga baseia-se no uso de inseticidas, principalmente o endosulfam, mas a sua utilização intensiva e repetida leva ao desenvolvimento de resistência do inseto ao produto, além de causar problemas ambientais e contaminação dos alimentos e agricultores (Neves \& Hirose 2005). A contínua utilização do controle químico de insetos da agricultura com agrotóxicos não seletivos provoca desequilíbrios, como a eliminação de insetos benéficos, explosões populacionais de pragas e, principalmente, a perda da eficácia de inseticidas mediante a seleção de populações resistentes aos compostos químicos repetidamente utilizados (Kay \& Collins 1987).

A busca por novos inseticidas constitui-se num campo de investigação amplo. A grande variedade de substâncias presentes na flora continua sendo um enorme atrativo na área de controle de insetos (Vieira \& Fernandes 1999). Estes produtos de origem botânica desempenham papel na interação da planta com o ambiente, como defesa contra herbivoria e patógenos, competição entre plantas e atração de organismos benéficos, como polinizadores, dispersores de sementes e microrganismos simbiontes (Raven et al. 2001, Castro et al. 2005). Pesquisas com plantas inseticidas são realizadas com o objetivo de se descobrir moléculas com atividade contra insetos que permitam a síntese de novos produtos inseticidas e a obtenção de inseticidas naturais para o uso direto no controle de insetos-praga (Vendramim 2000).

Recentemente, o interesse na identificação de novos compostos em espécies do gênero Piper intensificou-se devido à detecção de diversas substâncias biologicamente ativas. Este gênero caracteriza-se pela produção de classes típicas de compostos, tais como amidas, ácidos benzóicos, lignanas e alcalóides (Lago et al. 2004). A piperina foi a primeira amida a ser isolada de espécies de Piper, a qual tem maior atividade inseticida do que as piretrinas (Estrela et al. 2003), também descobertas em plantas e amplamente comercializadas no mundo (Soderlund et al. 2002).

Piper hispidum Kunth distribui-se pela América Central, Antilhas e América do Sul. No Brasil, ocorre nos estados do Amazonas, Pará, Piauí, Ceará, Pernambuco, Bahia, Rio de Janeiro, Paraná, Santa Catarina, Mato Grosso, São Paulo e Mato do Grosso do Sul (Guimarães
\& Giordano 2004), sendo conhecida popularmente pelos nomes jaborandi ou falso-jaborandi, e tem seu uso popular no combate a dores estomacais e afecções de pele (Navickiene et al. 2000).

Potzernheim et al. (2006) identificaram 26 compostos no óleo essencial de $P$. hispidum, sendo que destes $23,0 \%$ são monoterpenos não oxigenados, $11,0 \%$ monoterpenos oxigenados, $15,4 \%$ sesquiterpenos não oxigenados e $34,6 \%$ sequiterpenos oxigenados. Os constituintes encontrados em maior quantidade foram: $\beta$-pineno (19,7\%), $\alpha$-pineno $(9,0 \%), \delta$-3 careno $(7,4 \%)$, $\alpha$-cadinol (6,9\%) e espatulenol $(6,2 \%)$.

Considerando que os compostos químicos desta espécie ainda não foram estudados como inseticidas, este trabalho teve como objetivo avaliar o efeito do extrato de folhas de $P$. hispidum sobre $H$. hampei por aplicação tópica, superfície contaminada e efeito de repelência.

\section{Material e métodos}

A planta e preparação do extrato - As folhas de Piper hispidum Kunth (Piperaceae) foram coletadas no câmpus da Universidade Federal de Rondônia, em Porto Velho, RO, em abril de 2006. A identificação botânica da espécie foi realizada pelo Dr. José Gomes do Herbário do Instituto Nacional de Pesquisa da Amazônia (Inpa), onde uma exsicata encontra-se depositada sob o número 216630.

A extração foi realizada a partir de folhas devidamente secas e trituradas $(1,2 \mathrm{~kg})$, utilizando-se acetona $(3 \mathrm{~L})$, à temperatura ambiente. O solvente foi destilado sob pressão reduzida, resultando em 21,0 g de uma massa de coloração marrom escuro.

Insetos - Os insetos adultos de Hypothenemus hampei (Ferrari, 1867 - Coleoptera: Scolytidae) utilizados foram oriundos da criação estoque da Embrapa Rondônia. Os insetos foram coletados em frutos maduros de café, armazenados em caixas de madeira $(26 \times 37 \times 13 \mathrm{~cm})$ (umidade $45 \%)$, sendo que cada caixa recebeu 350 a 400 frutos brocados, preenchendo aproximadamente metade do volume da caixa. Esses insetos foram mantidos em gaiolas com tela de filó, à temperatura de $25 \pm 2{ }^{\circ} \mathrm{C}$. Para evitar contaminações fúngicas e possíveis microorganismos entomopatogênicos aderidos aos frutos, estes foram lavados com água corrente. Posteriormente, para eliminar o excesso de umidade, foram deixados para secar à sombra por 48 horas, sendo devolvidos para a gaiola.

Exposição por aplicação tópica - No Laboratório de Entomologia da Embrapa Rondônia, os insetos adultos foram borrifados com $0,25 \mathrm{~mL}$ das diluições do extrato em acetona a 25,0;5,0;0,1;0,02;0,004;0,0008 $\mathrm{mg} \mathrm{mL}^{-1}$, utilizando-se acetona como controle. Em seguida, foram transferidos para placas de Petri de 9,0 $\mathrm{cm}$ de diâmetro, cobertas com papel filtro. Utilizou-se dez insetos por placa, em delineamento inteiramente casualizado com quatro repetições. Avaliou-se a 
mortalidade dos insetos nas 48 horas seguintes à pulverização. $\mathrm{Na}$ avaliação da mortalidade dos insetos, foram considerados vivos todos os insetos que moviam qualquer parte do corpo quando estimulados. Os dados foram submetidos à análise de variância e as médias comparadas pelo teste de Tukey.

Exposição por superfície contaminada - Foram utilizadas as mesmas soluções do bioensaio com aplicação tópica. Adicionou-se 1,0 mL destas soluções a placas de Petri de 9,0 cm de diâmetro, contendo papel de filtro tipo Whatman no 01 . Foram colocados dez insetos por placa, em quatro repetições, em delineamento inteiramente casualizado, avaliando-se a mortalidade dos insetos durante as 48 horas seguintes. Os dados foram submetidos à análise de variância e as médias comparadas pelo teste de Tukey.

Efeito de repelência - Frutos maduros de café sadio foram imersos em solução de extrato acetônico a $0,5 \mathrm{mg} \mathrm{mL}^{-1}$, por um minuto; utilizando-se acetona como controle. Placas de Petri de 9,0 cm de diâmetro foram demarcadas com uma linha central, sendo colocados três frutos de café tratados com solução de extrato de um lado e três frutos do grupo controle do outro lado. Na região central da placa, foram inseridos 10 insetos. Foi avaliado o número de insetos que se deslocaram para cada um dos lados de cada placa, durante os quinze minutos seguintes. O delineamento foi inteiramente casualizado com dez insetos por tratamento e dez repetições (placas). O índice de repelência (RI) foi calculado como indicado por Villalobos \& Robledo (1998), RI $=(\mathrm{C}-\mathrm{T}) /(\mathrm{C}+\mathrm{T})$ $\times 100$, onde $(\mathrm{C})$ é o número de insetos que dirigiram-se para a área onde havia a dieta controle e $(\mathrm{T})$ o número de insetos que dirigiram-se para a área onde havia dieta contendo solução de extrato. Os dados foram submetidos à análise de variância e as médias comparadas pelo teste de Tukey.

\section{Resultados e discussão}

Na tabela 1 estão apresentados os resultados do bioensaio em superfície contaminada, realizado com insetos de $H$. hampei, em relação a diferentes diluições do extrato de $P$. hispidum, em um período de 48 horas. Os resultados mostram que o extrato utilizado foi bastante eficiente na indução da mortalidade dos insetos e abre novas perspectivas quanto à sua utilização como inseticida no controle biológico de pragas.

Após24horas debioensaio, a diluição de $25,0 \mathrm{mg} \mathrm{mL}^{-1}$ promoveu $100 \%$ de mortalidade. As diluições de 5,0 a $0,004 \mathrm{mg} \mathrm{mL}^{-1}$ também foram eficientes, sendo que apenas a diluição de $0,0008 \mathrm{mg} \mathrm{mL}^{-1}$ não apresentou efeito significativo em relação ao controle. Em termos gerais, os resultados obtidos após 24 horas não diferiram muito daqueles observados após 48 horas de exposição, evidenciando a rapidez da ação inseticida do extrato. É importante ressaltar que a velocidade com que o extrato provoca mortalidade pode ser de grande relevância, uma
Tabela 1. Efeito do extrato de folhas de Piper hispidum sobre Hypothenemus hampei, em superfície contaminada, durante 24 e 48 horas. Letras diferentes correspondem a diferenças significativas dentro de cada coluna $(P=0,05)$.

Table 1. Effect of Piper hispidum leaves extract on Hypothenemus hampei by contaminated surface during 24 and 48 hours. Different letters mean statistically significant differences within each collumn $(P=0.05)$.

Taxa de mortalidade de H. hampei*

Diluições do extrato $(\%)$

\begin{tabular}{lcc}
\cline { 2 - 3 } & 24 horas & 48 horas \\
\hline $25,0 \mathrm{mg} \mathrm{mL}^{-1}$ & $100^{\mathrm{a}}$ & $100^{\mathrm{a}}$ \\
$5,0 \mathrm{mg} \mathrm{mL}^{-1}$ & $80^{\mathrm{b}}$ & $80^{\mathrm{b}}$ \\
$0,1 \mathrm{mg} \mathrm{mL}^{-1}$ & $75^{\mathrm{b}}$ & $80^{\mathrm{b}}$ \\
$0,02 \mathrm{mg} \mathrm{mL}^{-1}$ & $50^{\mathrm{c}}$ & $65^{\mathrm{c}}$ \\
$0,004 \mathrm{mg} \mathrm{mL}^{-1}$ & $45^{\mathrm{c}}$ & $50^{\mathrm{d}}$ \\
$0,0008 \mathrm{mg} \mathrm{mL}^{-1}$ & $5^{\mathrm{d}}$ & $5^{\mathrm{e}}$ \\
Controle & $5^{\mathrm{d}}$ & $5^{\mathrm{e}}$ \\
\hline
\end{tabular}

vez que, em condições de campo, o contato do inseto com o extrato pode ser relativamente rápido.

Resultados semelhantes foram obtidos por Fazolin et al. (2005), avaliando o efeito do óleo essencial de Piper aduncum L. sobre insetos adultos de Cerotoma tingomarianus (Bechyné) em superfície contaminada, nas concentrações de $10,1,10^{-1}, 10^{-2}, 10^{-3}, 10^{-4} \mathrm{e} 10^{-5} \mathrm{mg} \mathrm{mL}^{-1}$, obtendo-se praticamente $100 \%$ de mortalidade com a maior concentração, $10 \mathrm{mg} \mathrm{mL}^{-1}$, após 48 horas.

Por outro lado, concentrações muito mais altas foram necessárias para atingir $100 \%$ de mortalidade no estudo realizado por Estrela et al. (2006), que observaram o efeito inseticida dos óleos essenciais de folhas de Piper hispidinervum C. DC. e Piper aduncum sobre Sitophilus zeamais Motsch. em superfície contaminada, nas concentrações de 300, 200, 100, 75, 50, 25 e $10 \mathrm{mg} \mathrm{mL}^{-1}$. Porcentagens de mortalidade próximas a $100 \%$ só foram atingidas com a concentração de $300 \mathrm{mg} \mathrm{mL}^{-1}$. A concentração de $200 \mathrm{mg} \mathrm{mL}^{-1}$ resultou em aproximadamente 78 e $63 \%$ de mortalidade, para $P$. aduncum e $P$. hispidinervum, respectivamente.

O trabalho de Flores et al. (1993) também envolveu concentrações altas em comparação com o presente estudo. Os autores testaram o extrato de Piper nigrum no controle do caruncho Zabrotes subfasciatus (Bohemann) em sementes armazenadas de Phaseolus vulgaris L., e sugeriram ser a piperina o principal princípio ativo que teria efeito inseticida, com efeito residual de até 90 dias, nas concentrações de 200 a $900 \mathrm{mg} \mathrm{mL}^{-1}$. 
Na tabela 2 estão apresentados os resultados do bioensaio com aplicação tópica, realizado com insetos de H. hampei, em relação a diferentes diluições do extrato de $P$. hispidum, dentro de um período de 48 horas. Comparando-se as tabelas 1 e 2, pode-se observar que, de forma geral, a aplicação em superfície contaminada foi mais eficiente que a aplicação tópica. A aplicação tópica também foi eficiente na promoção da mortalidade dos insetos, mas não atingiu $100 \%$, como ocorreu na aplicação em superfície contaminada. Provavelmente, isto está relacionado à capacidade de retenção do papel de filtro, que proporcionou um contato mais prolongado dos insetos com as diluições do extrato. A aplicação tópica promoveu até $65 \%$ de mortalidade na diluição de $25,0 \mathrm{mg} \mathrm{mL}^{-1}$. As diluições do extrato $25,0,5,0 \mathrm{e}$ $0,1 \mathrm{mg} \mathrm{mL}^{-1}$ apresentaram eficiência e não diferiram entre si significativamente. As diluições de 0,02; 0,004 e $0,0008 \mathrm{mg} \mathrm{mL}^{-1}$ não diferiram significativamente do controle do experimento.

Resultados bastante inferiores foram obtidos no estudo realizado por Fazolin et al. (2005). Estes autores avaliaram o efeito do óleo essencial de Piper aduncum sobre insetos adultos de Cerotoma tingomarianus por aplicação tópica, testando as diluições de 10, 25, 50, 75, 100,200 e $300 \mathrm{mg} \mathrm{mL}^{-1}$, que resultaram em mortalidade de 5 a $30 \%$ dos insetos.

Estrela et al. (2006) observaram o efeito do contato tópico dos óleos essenciais de folhas de Piper hispidinervum e Piper aduncum sobre Sitophilus

Tabela 2. Efeito do extrato de folhas de Piper hispidum sobre Hypothenemus hampei, em aplicação tópica, durante 24 e 48 horas. Letras diferentes correspondem a diferenças significativas dentro de cada coluna $(P=0,05)$.

Table 2. Effect of Piper hispidum leaves extract on Hypothenemus hampei by topical application during 24 and 48 hours. Different letters mean statistically significant differences within each collumn $(P=0.05)$.

Taxa de mortalidade de H. hampei*

Diluições do extrato

$(\%)$

\begin{tabular}{lcc}
\cline { 2 - 3 } & 24 horas & 48 horas \\
\hline $25,0 \mathrm{mg} \mathrm{mL}^{-1}$ & $55^{\mathrm{a}}$ & $65^{\mathrm{a}}$ \\
$5,0 \mathrm{mg} \mathrm{mL}^{-1}$ & $60^{\mathrm{a}}$ & $60^{\mathrm{a}}$ \\
$0,1 \mathrm{mg} \mathrm{mL}^{-1}$ & $55^{\mathrm{a}}$ & $60^{\mathrm{a}}$ \\
$0,02 \mathrm{mg} \mathrm{mL}^{-1}$ & $20^{\mathrm{b}}$ & $25^{\mathrm{b}}$ \\
$0,004 \mathrm{mg} \mathrm{mL}^{-1}$ & $20^{\mathrm{b}}$ & $25^{\mathrm{b}}$ \\
$0,0008 \mathrm{mg} \mathrm{mL}^{-1}$ & $15^{\mathrm{b}}$ & $25^{\mathrm{b}}$ \\
Controle & $20^{\mathrm{b}}$ & $20^{\mathrm{b}}$ \\
\hline
\end{tabular}

zeamais. Os autores testaram as concentrações de 300 , $200,100,75,50$ e $25 \mathrm{mg} \mathrm{mL}^{-1}$. Constatou-se que valores de mortalidade acima de $70 \%$ somente foram alcançados nas concentrações acima de as concentrações acima de 100 e $200 \mathrm{mg} \mathrm{mL}^{-1}$ para os óleos de $P$. hispidinervum e $P$. aduncum, respectivamente.

Scott et al. (2005) testaram o efeito de inseticidas botânicos sobre o escaravelho europeu, Rhizotrogus majalis (Razoumowsky), conhecido como praga dos gramados, e obtiveram efeito positivo (mortalidade significativamente superior à observada no controle do experimento) com a aplicação de extrato de Piper nigrum em condições de campo (aplicado diretamente sobre gramados infestados), na concentração de $20 \mathrm{mg} \mathrm{mL}^{-1}$ para controle do segundo e terceiro instar. Em um segundo experimento, foi necessária a aplicação de $40 \mathrm{mg} \mathrm{mL}^{-1}$ para causar mortalidade significativa em insetos mais velhos do terceiro instar.

O extrato de sementes de Piper guineense Schumach. foi estudado por Ashamo \& Odeyemi (2001) no controle de Sitophilus zeamais aplicado topicamente a grãos de milho (Zea mays L.). Os autores observaram $90 \%$ de mortalidade dos insetos, com a aplicação de $30 \mathrm{mg} \mathrm{mL}^{-1}$ do extrato.

No bioensaio de repelência, em termos gerais, não foi obtido resultado positivo (tabela 3 ). As avaliações deste bioensaio, realizadas a $1,5,10$ e 15 minutos, resultaram em índices de repelência de 20,0;30,6; 36,0 e 32,0, respectivamente. De acordo com Villalobos \& Robledo (1998), podem ser consideradas repelentes substâncias cujo índice está acima de 50 . No presente estudo, este bioensaio demonstrou que o extrato de

Tabela 3. Efeito de repelência do extrato de folhas de Piper hispidum sobre Hypothenemus hampei. Porcentagens de insetos que se deslocaram para a área onde havia a dieta controle ou para a dieta com extrato $\left(0,5 \mathrm{mg} \mathrm{mL}^{-1}\right)$; ou permaneceram na região central (neutra); e Índices de Repelência obtidos, durante 15 minutos.

Table 3. Repellence effect of Piper hispidum leaves extract on Hypothenemus hampei. Percentages of insects that moved to the area with the control diet or to the diet with extract $\left(0.5 \mathrm{mg} \mathrm{mL}^{-1}\right)$; or remained in the central region (neutral); and obtained Repellence Index, during 15 minutes.

\begin{tabular}{|c|c|c|c|c|}
\hline \multirow{2}{*}{$\begin{array}{c}\text { Tempo } \\
\text { (minutos) }\end{array}$} & Controle & Extrato & Região neutra & \multirow{2}{*}{$\begin{array}{l}\text { Índice de } \\
\text { repelência }\end{array}$} \\
\hline & \multicolumn{3}{|c|}{$(\%)$} & \\
\hline 1 & 48 & 32 & 20 & 20,0 \\
\hline 5 & 64 & 34 & 2 & 30,6 \\
\hline 10 & 68 & 32 & 0 & 36,0 \\
\hline 15 & 66 & 34 & 0 & 32,0 \\
\hline
\end{tabular}


folha de $P$. hispidum não é eficiente como repelente para H. hampei.

Os resultados do presente trabalho evidenciam a ação inseticida do extrato das folhas de $P$. hispidum contra $H$. hampei em condições de laboratório, sugerindo seu potencial no controle deste inseto-praga em condições de campo. Entretanto, são necessários estudos para identificar efeitos tóxicos deste extrato contra outros organismos, como peixes e outros insetos (inimigos naturais de $H$. hampei, como Cephalonomia sp., a vespa da Costa do Marfim), como é o caso de P. piscatorum, conhecida por suas propriedades piscicidas e inseticidas (McFerren \& Rodriguez, 1999), além de microrganismos, tais como o fungo Beauveria bassiana (Balsamo) Vuillemin, atualmente utilizado no controle biológico de H. hampei.

Agradecimentos - Os autores agradecem ao Conselho Nacional de Desenvolvimento Científico e Tecnológico (CNPq), pela concessão de Bolsa de Iniciação Científica a Renato Abreu Lima (processo no 109168/2006-2), e ao Dr. José Gomes do Herbário do Instituto Nacional de Pesquisa da Amazônia (Inpa), pela identificação botânica.

\section{Referências bibliográficas}

ASHAMO, M.O. \& ODEYEMI, O.O. 2001. Protection of maize against Sitophilus zeamais Motsch. using seed extracts from some indigenous plants. Zeitschrift für Pflanzenkrankheiten und Pflanzenschutz 108:320-327.

BANDEIRA, R.D.C.C., TOCI, A.T., TRUGO, L.C. \& FARAH, A. 2009. Composição volátil dos defeitos intrínsecos do café por CG/EM-headspace. Química Nova 32:309-314.

CASTRO, P.R.C., KLUGE, R.A. \& PERES, L.E.P. 2005. Manual de fisiologia vegetal. Ceres, São Paulo.

ECCARDI, F. \& SANDALJ, V. 2000. O café - ambientes e diversidade. Casa da Palavra, Alfenas.

ESTRELA, J.L.V., GUEDES, R.N.C., MALTHA, C.R.A. \& FAZOLIN, M. 2003. Toxicidade de amidas análogas à piperina a larvas de Ascia monuste orseis Godart (Lepidoptera: Pieridae) e Spodoptera frugiperda (J.E. Smith) (Lepidoptera: Noctuidae). Neotropical Entomology 32:343-346.

ESTRELA, J.L.V., FAZOLIN, M., CATANI, V., ALÉCIO, M.R. \& LIMA, M.S. 2006. Toxicidade de óleos essenciais de Piper aduncum e Piper hispidinervum em Sitophilus zeamais. Pesquisa Agropecuária Brasileira 41:217-222.

FAZOLIN, M., ESTRELA, J.L.V., CATANI, V., LIMA, M.S. \& ALÉCIO, M.R. 2005. Toxicidade do óleo de Piper aduncum L. a adultos de Cerotoma tingomarianus Bechyné (Coleoptera: Chrysomelidae). Neotropical Entomology 34:485-489.
FLORES, W.L., SAMPAIO, L.S.V., MARQUES, O.M. \& COSTA, J.A. 1993. Efeitos dos extratos de pimenta do reino e cinamomo e do malation no controle do caruncho Zabrotes subfasciatus em sementes de feijão (Phaseolus vulgaris) armazenadas. Insecta 2:11-22.

GUIMARÃES, E.F. \& GIORDANO, L.C.S. 2004. Piperaceae do nordeste brasileiro I: Estado do Ceará. Rodriguesia 55:21-46.

KAY, I.R. \& COLLINS, P.J. 1987. The problem of resistance to insecticides in tropical insects pests. Insect Science Apllicata 8:715-721.

LAGO, J.H., RAMOS, C.S., CASANOVA, D.C.C., MORANDIM, A.A., BERGAMO, D.C.B., CAVALHEIRO, A.J., BOLZANI, V.S., FURLAN, M., GUIMARÃES, E.F., YOUNG, M.C.M. \& KATO, M.J. 2004. Benzoic acid derivatives from Piper species and their fungitoxic activity against Cladosporium cladosporioides and C. sphaerospermum. Journal of Natural Products 67:1783-1788.

MCFERREN, M.A. \& RODRIGUEZ, E. 1998. Piscicidal properties of piperovatine from Piper piscatorum (Piperaceae). Journal of Ethnopharmacology 60:183187.

NAJBERG, S. \& PEREIRA, R.O. 2004. Novas estimativas do modelo de geração de empregos do BNDES. Sinopse Econômica 133:25-32.

NAVICKIENE, H.M.D., ALÉCIO, A.C., KATO, M.J., BOLZANI, V.S., YOUNG, M.C.M., CAVALHEIRO, A.J. \& FURLAN, M. 2000. Antifungal amides from Piper hispidum and Piper tuberculatum. Phytochemistry 55:621-626.

NEVES, P.M.O.J. \& HIROSE, E. 2005. Seleção de isolados de Beauveria bassiana para o controle biológico da broca-do-café, Hypothenemus hampei (Ferrari) (Coleoptera: Scolytidae). Neotropical Entomology 34:77-82.

POTZERNHEIM, M.C.L., BIZZO, H.R. \& VIEIRA, R.F. 2006. Análise dos óleos essenciais de três espécies de Piper coletadas na região do Distrito Federal (Cerrado) e comparação com óleos de plantas procedentes da região de Paraty, RJ (Mata Atlântica). Revista Brasileira de Farmacognosia 16:246-251.

RAVEN, F.H., EVERT, R.T. \& CURTIS, H. 2001. Biologia vegetal. Guanabara, Rio de Janeiro.

RESENDE, M.D.V., FURLANI-JÚNIOR, E., MORAES, M.L.T. \& FAZUOLI, L.C. 2001. Estimativas de parâmetros genéticos e predição de valores genotípicos no melhoramento do cafeeiro pelo procedimento REML/BLUP. Bragantia 60:185-193.

SCOTT, I.M., GAGNON, N., LESAGE, L., PHILOGE, B.J.R. \& ARNASON, T. 2005. Efficacy of botanical insecticides from Piper species (Piperaceae) extracts for control of european chafer (Coleoptera: Scarabaeidae). Journal of Economic Entomology 98:845-855. 
SODERLUND, D.M., CLARK, J.M., SHEETS, L.P., MULLIN, L.S., PICCIRILLO, V.J., SARGENT, D., STEVENS, J.T. \& WEINER, M.L. 2002. Mechanisms of pyrethroid neurotoxicity: implications for cumulative risk assessment. Toxicology 171:3-59.

TASSARO, H. 2008. Café - História e perspectivas. http:// www.bicafe.com.br (acesso em 20/05/2008).

VEGRO, C.L.R., PINO, F.A., NOGUEIRA JÚNIOR, S. \& MORICOCHI, L. 2005. Do café verde ao café torrado e moído: vantagens e dificuldades na exportação. Revista Brasileira de Comércio Exterior 84:60-71.
VENDRAMIM, J.D. 2000. Plantas inseticidas e controle de pragas. Informativo da Sociedade Entomológica do Brasil 25:1-5.

VIEIRA, C.P. \& FERNANDES, B.J. 1999. Plantas inseticidas. In Farmacognosia - da planta ao medicamento (C.M. Simões, E.P. Schenkel, G. Gosmann, J.C.P. Mello, L.A. Mentz \& P.R. Petrovick, orgs.). UFRGS/UFSC, Porto Alegre, p.739-754.

VILLALOBOS, M.J.P. \& ROBLEDO, A. 1998. Screening for anti-insect activity in Mediterranean plants. Industrial Crops and Products 8:183-194. 